\title{
The new Heavy Ion ERDA set up at iThemba LABS Gauteng: Multilayer thin film depth profiling using direct calculation and Monte Carlo simulation codes $\mathrm{M}$.
}

\author{
Msimanga ${ }^{\mathrm{a}, *}$, D. Wamwangi $^{\mathrm{b}}$, C.M. Comrie ${ }^{\mathrm{c}}$, C.A. Pineda-Vargas ${ }^{\mathrm{c}}$, M. Nkosi $^{\mathrm{c}}$, T. Hlatshwayo $^{\mathrm{d}}$ \\ a iThemba LABS Gauteng, Private Bag 11, WITS 2050, Johannesburg, South Africa \\ ${ }^{\mathrm{b}}$ School of Physics, University of the Witwatersrand, Johannesburg, South Africa \\ ${ }^{\mathrm{c}}$ Materials Research Department, iThemba LABS, P.O. Box 722, Somerset West, Cape Town, South Africa ${ }^{\mathrm{d}}$ Physics Department, University of Pretoria, Pretoria, South Africa \\ ${ }^{*}$ Corresponding author. Tel.: +27 11351 7026; fax: +27 11351 7053. E-mail address: mandla@tlabs.ac.za (M. Msimanga).
}

\section{A B S T R A C T}

We report here on the recently built Heavy Ion ERDA set up at iThemba LABS Gauteng; describing a typ-ical application in the study of interfacial reactions in an $\mathrm{Al}_{2} \mathrm{O} 3-\mathrm{Ti}$ ceramic-metal multilayer structure annealed in vacuum at $800{ }^{\circ} \mathrm{C}$ for $2 \mathrm{~h}$. Depth profile extraction was found to be best obtained through combined use of direct calculation and Monte Carlo simulation codes as opposed to using just either of the methods. The obtained profile suggests a case of the Kirkendall effect, whereupon the inter-diffu-sion between the metal and the ceramic was largely due to the faster diffusion of the metal into the amor-phous ceramic than diffusion of the ceramic elements into the metallic layer.

Keywords:

Heavy Ion ERDA

Energy resolution

Multiple scattering

Depth profiling

Kirkendall effect

\section{Introduction}

Thin film materials are increasingly finding usage in more and more complex structures of technological importance at the micro-/nano-metre length scale. They are used in a multitude of applications ranging from absorptive coatings in optical and solar energy devices to protective coatings for biomaterials, and a host of other device applications in the semiconductor industry [1-3]. The optical, mechanical, and electrical properties of thin film structures depend a lot on film composition, interfacial topography and depth profile of the layer stack. Of the vast number of surface and sub-surface analysis techniques available to material scientists, nuclear techniques can readily offer quantitative and standard-free analysis with high resolution depth profiling not easily attainable by other means [4-6]. Heavy Ion Elastic Recoil Detection Analysis (Heavy Ion ERDA) is one of the latest nuclear analytical techniques whose development has been spurred on mostly by the need for simultaneous analysis of light elements in heavy element matrices $[4,7,8]$. When built with a mass dispersive recoil detector system, it is possible with Heavy Ion ERDA to obtain depth profiles of all target elements present in a layer structure in a single measurement.

The conversion of elemental energy spectra into depth profiles is generally done through either direct calculation algorithms like KONZERD [9] or through spectrum simulation codes. Simulation codes generally fall into one of two categories; analytical like SIM-NRA [10] and NDF [11] or Monte Carlo based like CORTEO [12] and MCERD [13]. Analysts, in their reports, invariably describe depth profiles extracted from energy spectra using either of the methods but hardly both [14-17]. It is widely accepted that both methods have relative advantages and disadvantages over each other [18]. For example, whereas the direct calculation method will only provide information from the sample, Monte Carlo simulations may include phantom structural details that lead to a good fit to experimental data but not necessarily reflecting the true sample structure.

On the other hand, the energy resolution in raw energy spectra used in the direct calculation method is a convolution of the energy resolution due to the detector system and due to geometrical and energy straggling and multiple scattering effects, all of which are not taken into account when calculating the depth profiles. Monte Carlo simulation, by calculating individual ion trajectories, takes these effects into account in a natural way. It is only logical then that to get the best of both worlds, especially when the energy resolution $\Delta E$ of detector system is not of an ultra-high resolution type (i.e. $\Delta E$ not less than $1 \%$ ), both depth profiling methods should be used to complement each other to get optimal sample description. This is even more so when dealing with complex layer structures like multilayers with several interfaces. This article serves to introduce our Time of Flight (ToF) Heavy Ion ERDA facility to the ion beam analysis community, by describing an example of this suggested approach to extract elemental depth profiles from ToF ERDA data. 


\section{Experimental}

\subsection{The Heavy Ion ERDA set up}

Heavy Ion ERDA consists in the detection of recoil atoms knocked off the first few $100 \mathrm{~nm}$ of a target sample surface by a projectile beam coming in at a grazing incidence angle to the surface. Ion beam analysis at iThemba LABS Gauteng is centred on a 6 MV tandem accelerator. The ion source used for heavy ion production is an $860 \mathrm{C}$ sputter source with caesium gas as the source of sputtering ions. Energy calibration of the $6 \mathrm{MV}$ accelerator was carried out using two different reactions from which a weighted average of the $90^{\circ}$ bending magnet constant $(K)$ was obtained [19]. The first one was the ${ }^{27} \mathrm{Al}(\mathrm{p}, \mathrm{n})^{27} \mathrm{Si}$ reaction where the known sharp neutron emission threshold of $5.802 \pm 0.001 \mathrm{MeV}$ was used, and the second was the ${ }^{12} \mathrm{C}\left({ }^{16} \mathrm{O}, \alpha\right){ }^{24} \mathrm{Mg}^{*}$ reaction at $170^{\circ}$. The latter method entailed adjusting the incident beam energy until the en-ergy of the alpha particle reaction product for the ${ }^{24} \mathrm{Mg}^{*}$ level at $1.369 \mathrm{MeV}$ excitation was bracketed by the main peaks (5.443 MeV and 5.486 $\mathrm{MeV}$ ) from a ${ }^{241} \mathrm{Am}$ detector calibration source. The measured alpha energy was then used to infer the en-ergy of the incident ${ }^{16} \mathrm{O}^{5}$ ${ }^{+}$beam $[20]$.

At the core of our Heavy Ion ERDA set up is a mass dispersive ToF spectrometer consisting of two carbon foil based Microchannel Plate (MCP) time detectors a distance of $0.6 \mathrm{~m}$ apart, and a PIPS semiconductor energy detector at the end of the flight path just behind the second time detector. The spectrometer sits at $30^{\circ}$ to the incident beam direction. The total timing resolution of the spectrometer is typically less than 1.0 ns as shown in Fig. 1 for $12.6 \mathrm{MeV}$ ${ }^{16} \mathrm{O}$ recoils, where the resolution is $0.54 \mathrm{~ns}$. The signal processing electronics is made up of standard Nuclear Instruments Modules that include Constant Fraction Discriminators for fast tim-ing, a Time to Digital Converter and an Analogue to Digital Con-verter for the PIPS detector signal. The data acquisition system is based on the freely available MIDAS data acquisition platform [21], which was customised in-house for this set up.

Coincidence measurement of the ToF and energy of the recoil atoms, with the PIPS detector providing the trigger signal, leads to elemental separation according to mass. This results in 2-D scatter plots showing different curves (see Fig. 2), each corresponding to a particular atomic mass, from which elemental energy spectra can be extracted. The energy spectra are obtained through conversion of the ToF axis into a linear energy axis through re-binning of event by event energies calculated using the measured ToF for each

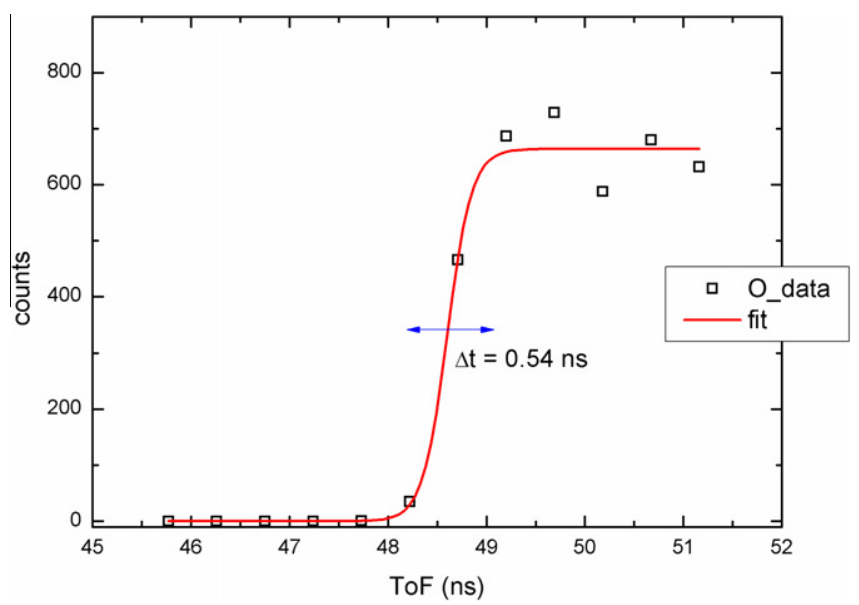

Fig. 1. Time of flight edge of $12.6 \mathrm{MeV}^{16} \mathrm{O}$ surface recoils from an $\mathrm{Al}_{2} \mathrm{O}_{3}$ layer on a $\mathrm{Si}$ substrate.
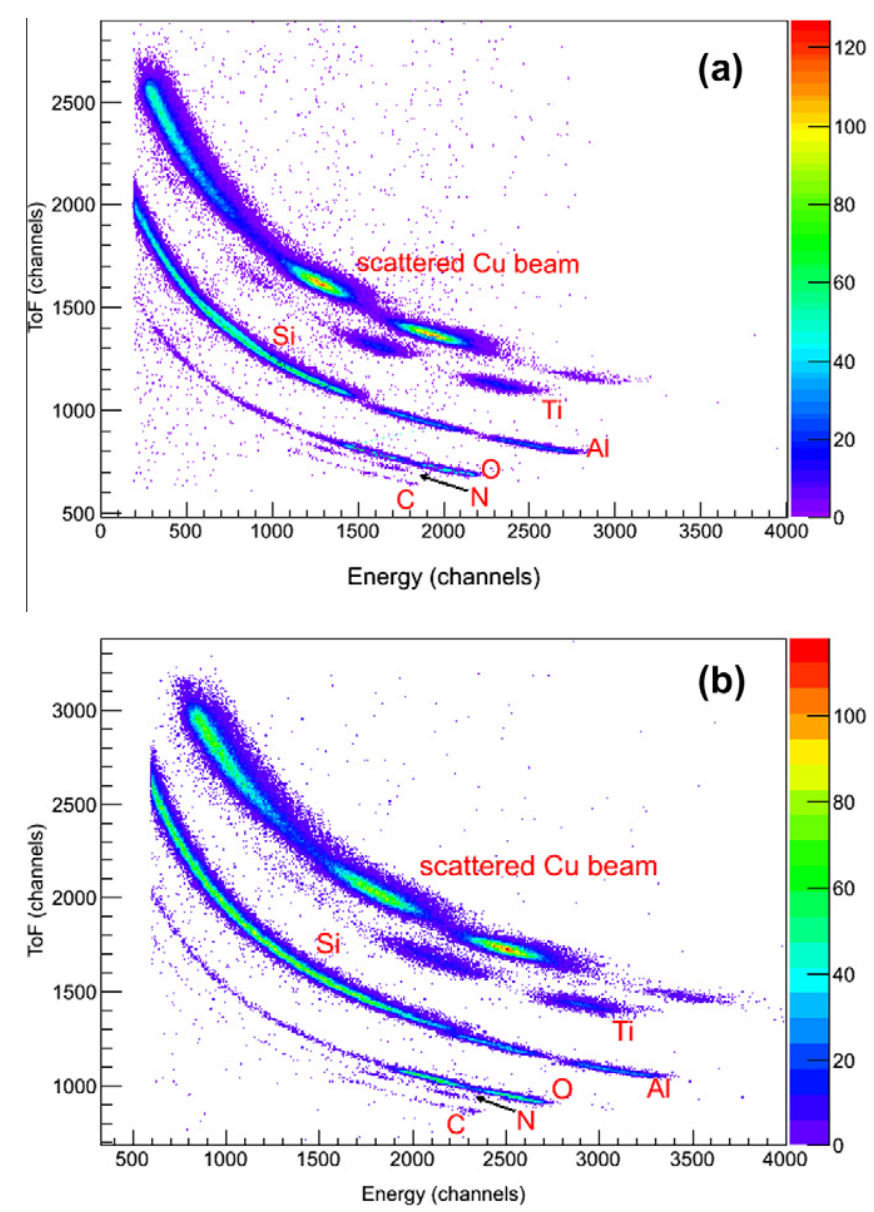

Fig. 2. ToF-Energy scatter plots of recoils (and forward scattered beam) from (a) the as-deposited and (b) the annealed samples.

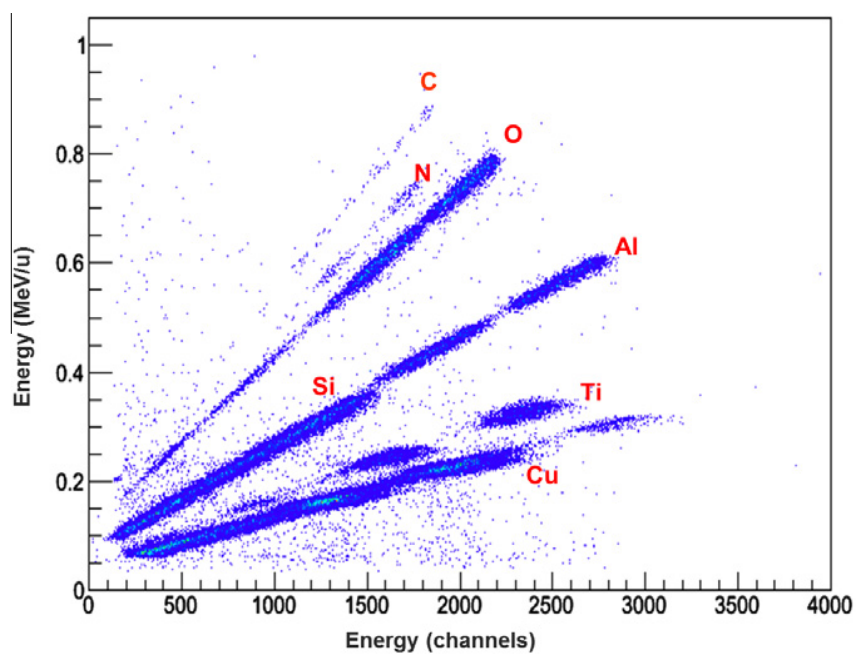

Fig. 3. A linear scatter plot of recoil ion energies from the as-deposited sample calculated from the measured ToF plotted as a function of the Si detector (trigger) response.

event as illustrated in Fig. 3. For depth analysis, elemental depth profiles are obtained through both direct calculation using KONZERD [9], and through iterative simulation of elemental ToF spectra using the Monte Carlo based code CORTEO [12]. 


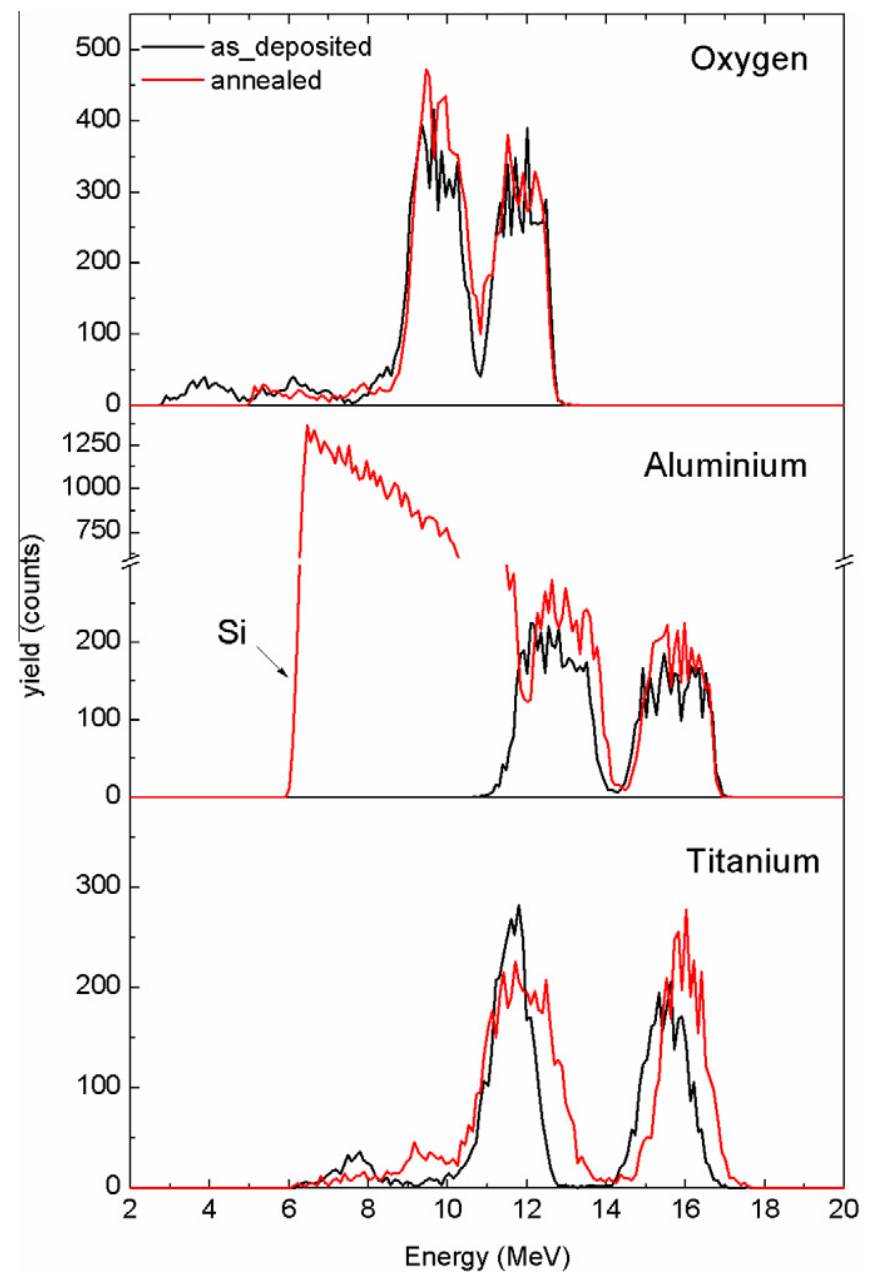

Fig. 4. Elemental energy spectra of recoils from the as-deposited and annealed samples.

\subsection{Target samples}

Two samples of bi-layer films of $\mathrm{Al}_{2} \mathrm{O}_{3}-\mathrm{Ti}$ on a Si substrate were chosen as test samples. The $\mathrm{Al}_{2} \mathrm{O}_{3}-\mathrm{Ti}$ interface has interesting properties that make it useful in a wide range of applications. In semiconductor packaging for example, solid state joints of $\mathrm{Al}_{2} \mathrm{O}_{3}$ fused with $\mathrm{Ti}$ exhibit high resistance to thermal stress due to the high thermal conductivity of the metal which facilitates stress relief when used as an interlayer [22,23]. Apart from the technological importance of the $\mathrm{Al}_{2} \mathrm{O}_{3}-\mathrm{Ti}$ interface, the nature of the interfacial reaction at elevated temperatures is still a contested issue [22-24], with different authors espousing different interpretations, hence the need for further investigation of the $\mathrm{Al}_{2} \mathrm{O}_{3}-\mathrm{Ti}$ bilayer system.

The samples were prepared simultaneously by electron beam deposition of alternate layers of $\mathrm{Ti}$ and $\mathrm{Al}_{2} \mathrm{O}_{3}$ onto $1.0 \mathrm{~cm}^{2} \mathrm{Si}$ substrates positioned edge-to-edge to ensure similar thickness depositions. The deposition was done at a base pressure of $10^{-6} \mathrm{mbar}$ with the substrate at room temperature. Rutherford Backscattering Spectrometry (RBS) measurements done in the past on different films deposited at one go on different substrates mounted on the same holder in our electron beam evaporator have shown no difference in the thickness of the deposited films. The nominal thickness of each $\mathrm{Al}_{2} \mathrm{O}_{3}-\mathrm{Ti}$ bi-layer, as determined by a quartz oscillator thickness monitor during deposition, was $20-50 \mathrm{~nm}$, respectively. The object of the analysis was to study through depth profiling the reaction mechanism between $\mathrm{Al}_{2} \mathrm{O}_{3}$ and Ti that eventually oc- curs at elevated temperatures for slow kinetics. To do this one sample was annealed in vacuum $\left(1 \times 10^{-6} \mathrm{mbar}\right)$ at $800^{\circ} \mathrm{C}$ for two hours after ramping it at $10^{\circ} \mathrm{C} / \mathrm{min}$ to the set point tempera-ture and a control sample left as-deposited. X-ray diffraction anal-ysis using Bragg-Brentano geometry showed no distinctive diffraction peaks in both samples except for the substrate peaks and so the films were presumed amorphous. Zalar and co-workers [24], who did a detailed study of the $\mathrm{Al}_{2} \mathrm{O}_{3}$-Ti interface, also report getting amorphous $\mathrm{Al}_{2} \mathrm{O}_{3}$ films on $\mathrm{Ti}$, though theirs were produced by sputter deposition.

\section{Measurements, results and discussion}

\subsection{ToF ERDA measurements}

For the ERDA measurement a $26.10 \mathrm{MeV}^{63} \mathrm{Cu}^{7+}$ beam was used, at a grazing incidence of $14^{\circ}$ to the sample surface. The beam current was set below $10 \mathrm{nA}$ for a run time of about $40 \mathrm{~min}$. Fig. 2a and b show 2-D ToF vs Energy scatter plots of recoil atoms (and the scattered $\mathrm{Cu}$ beam) from the as-deposited and the annealed films, respectively. The apparent difference in the channel ranges is due to the fact that the measurements were carried out at different times with different electronics settings (Time-to-Digital Converter range). That however, should be of no consequence to the analysis that follows since the energy per channel binning setting used was the same.

The atomic species present in the films are clearly separated according to mass. The gaps observed in the ${ }^{16} \mathrm{O},{ }^{27} \mathrm{Al}$ and ${ }^{48} \mathrm{Ti}$ mass lines are indicative of the different layers. Fig. 3 shows, as an example, the as-deposited film scatter plot now presented in the form of energy, calculated from the measured ToF, as a function of the PIPS detector response. It is evident from this plot that the slope of each contour line is dependent on the mass of the corresponding nuclide. A rough plot of atomic mass $(A)$ against slope $(X)$ for the known nuclides ${ }^{16} \mathrm{O},{ }^{28} \mathrm{Si}$ and ${ }^{63} \mathrm{Cu}$ was fitted with a function of
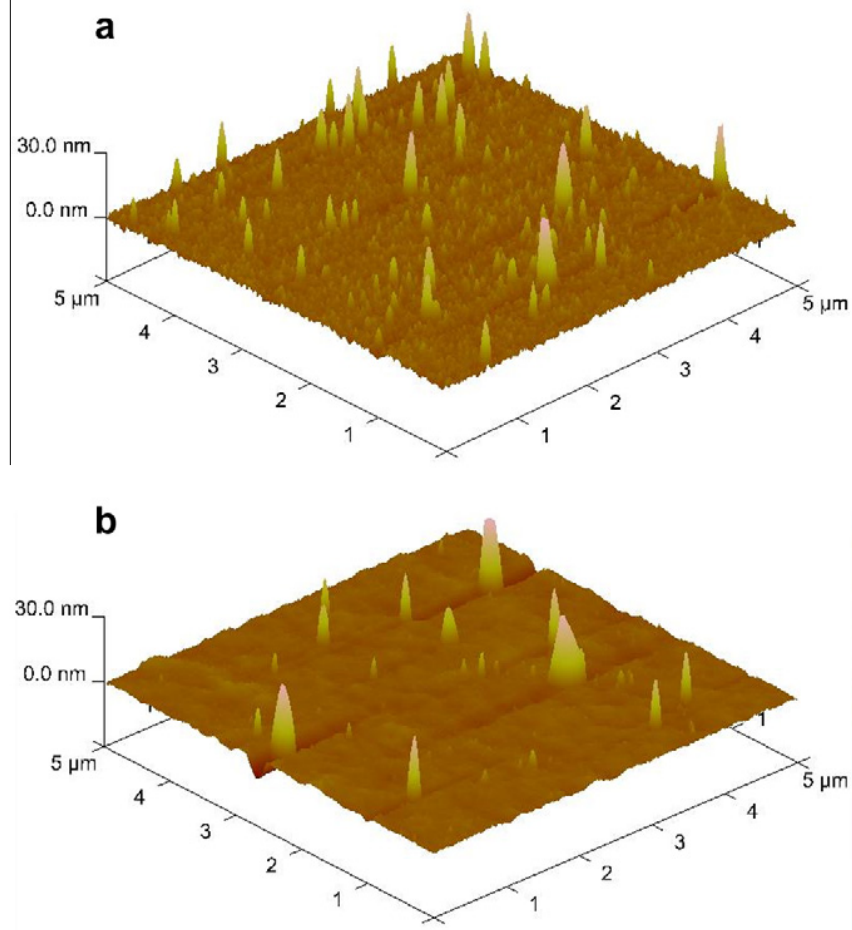

Fig. 5. Atomic Force Microscopy images of (a) virgin and (b) irradiated spots of the as deposited sample. 


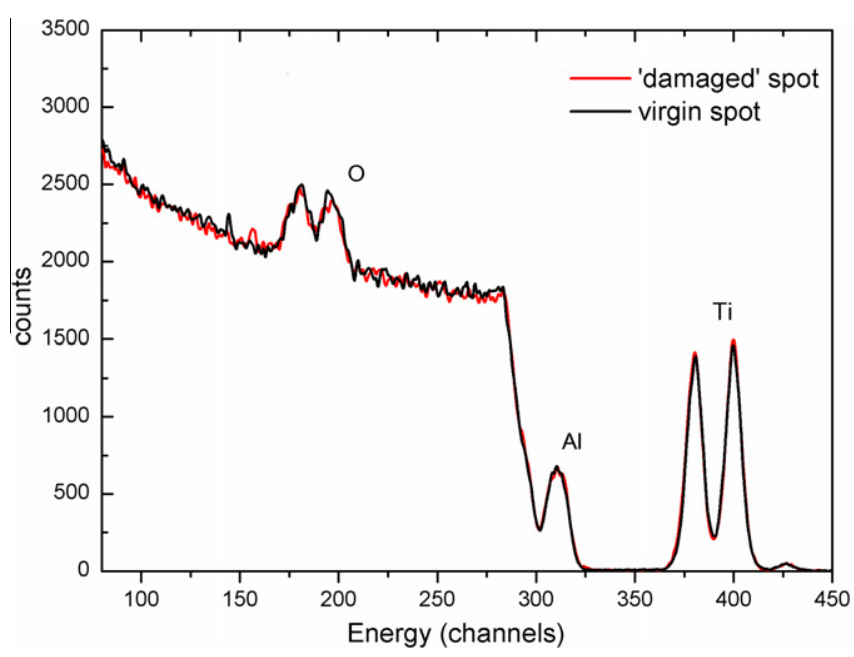

Fig. 6. RBS spectra from $\mathrm{Cu}$ irradiated (red) and virgin (black) regions of the asdeposited sample. (For interpretation of the references to colour in this figure legend, the reader is referred to the web version of this article.)

the form $A=k X^{b}$, where $k$ and $b$ are constants. For a sufficiently small mass range $b \approx 0$ and this observation was used to confirm the identity of the ${ }^{12} \mathrm{C}$ and ${ }^{14} \mathrm{~N}$ impurities using the slope of the ${ }^{16} \mathrm{O}$ contour line.

Two subtle points about the measurement are worth mentioning here. Firstly, the first "island" of the ${ }^{63} \mathrm{Cu}$ mass lines in Fig. 2 indicates presence of an impurity element heavier than ${ }^{48} \mathrm{Ti}$. This heavy element is also seen in the RBS spectra (channel number $\sim$ 425) shown later in Fig. 6. That there is no discernible corresponding mass line of recoils of this element in Fig. 2, in spite of the fact that the recoil cross section increases with the (square of the) atomic number of the target element, suggests that its relative content is insignificant. Secondly, hydrogen is a common surface impurity in thin film analysis [25], but could not be detected in this measurement. The detection efficiency of the ToF spectrometer for

${ }^{1} \mathrm{H}$ precluded its detection under the current measurement settings. In a separate measurement, the hydrogen detection efficiency was measured directly using a 1-2 MeV proton beam with the MCP detectors bias voltage set slightly above the recommended maximum and was found to be below 30\%. For heavier ions, from ${ }^{12} \mathrm{C}$ upwards, the ToF detector efficiency ranges from $90 \%$ to $95 \%$, and at much lower MCP bias voltages such as used in the current analyses. The end result of this is that the measured depth profiles are of relative atomic fractions; relative in the sense that not all the constituent target elements are included in the pro-file. The difference between the absolute and the relative profiles, however, should be negligible since the as yet unidentified heavy element is of quite low concentration and any (likely) hydrogen impurity is expected to be mostly surface contamination.

Elemental energy spectra for each sample were obtained by projecting each mass region in the $2 \mathrm{D}$ energy scatter plot onto the energy $(\mathrm{MeV} / \mathrm{u})$ axis and scaling the axis by the atomic mass of each nuclide. Fig. 4 shows superimposed energy spectra of ${ }^{16} \mathrm{O}$, ${ }^{27} \mathrm{Al}$ and ${ }^{48} \mathrm{Ti}$ recoils from both the as-deposited and annealed samples. There is no shift in the high energy edges of oxygen and aluminium recoils as expected, but only at lower energies, suggesting that interfacial reactions may have taken place. By far the most telling shift is the one observed in the Ti energy spectra, which strongly points to reactions (or Ti diffusion) taking place.

\subsection{Beam damage analysis}

A likely artefact in ion beam analysis, particularly in Heavy Ion ERDA, is the loss of thin film atoms due to ballistic effects during measurement [26]. This might lead to wrong thickness and depth profile conclusions if the beam damage is not insignificant. This effect is largely dependent on the combination of beam type, energy and dose, and the nature of the film under analysis. For example Trocellier and co-workers [27] report of degradation in the composition of a Kapton (polymide film) after bombardment with $50 \mathrm{MeV}$ ${ }^{32} \mathrm{~S}$ ions to fluences ranging from $2.8 \times 10^{13}$ to $1.3 \times 10^{14} \mathrm{ions} / \mathrm{cm}^{2}$. The degradation is attributed to a redistribution and loss of $\mathrm{C}, \mathrm{N}$ and $\mathrm{O}$ atoms in the polymer due to low binding energies character-istic of polymeric materials. In contrast, Whitlow et al. [28] report observing statistically insignificant changes in the stoichiometry of $\mathrm{SiO} 2 / \mathrm{Si}$ sample structures bombarded by $48 \mathrm{MeV}^{81} \mathrm{Br}$ ions up to a fluence of $2 \times 10^{14}$ ions $/ \mathrm{cm}^{2}$.

The fluence of the $26.1 \mathrm{MeV} \mathrm{Cu}$ beam used in the current analyses was in the order of $10^{12}$ ions $/ \mathrm{cm}^{2}$. Nonetheless it was decided to investigate if there was any material erosion accompanying the beam spot imprint left on the as-deposited sample. This beam spot was not immediately visible in the annealed sample. At first Atomic Force Microscopy (AFM) imaging was done to view possible changes in the surface topography, then Rutherford Backscattering Spectrometry (RBS) analyses were done on the ERDA beam spot and a selected undamaged region using $1.6 \mathrm{MeV} \mathrm{He}{ }^{+}$ions to check thickness changes.

Typical AFM images of virgin and irradiated regions of the asdeposited sample are shown in Fig $5 \mathrm{a}$ and b, respectively. The roughness was measured to be $1.2 \mathrm{~nm}$ for the virgin spot and $0.75 \mathrm{~nm}$ for the irradiated one. This suggests that one physical effect of the probing $\mathrm{Cu}-63$ beam was to smoothen the sample surface. The results of the RBS measurements done to check
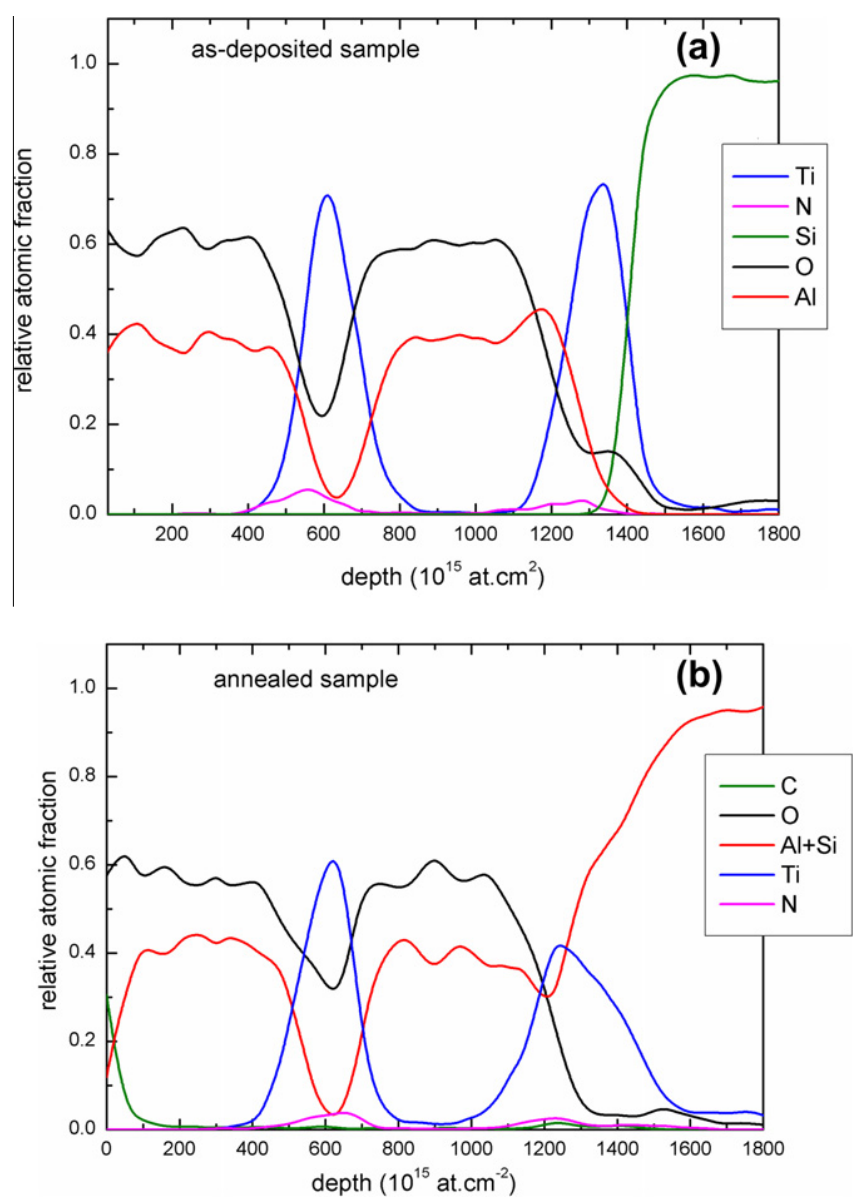

Fig. 7. KONZERD calculated depth profiles of (a) as-deposited and (b) annealed samples. 

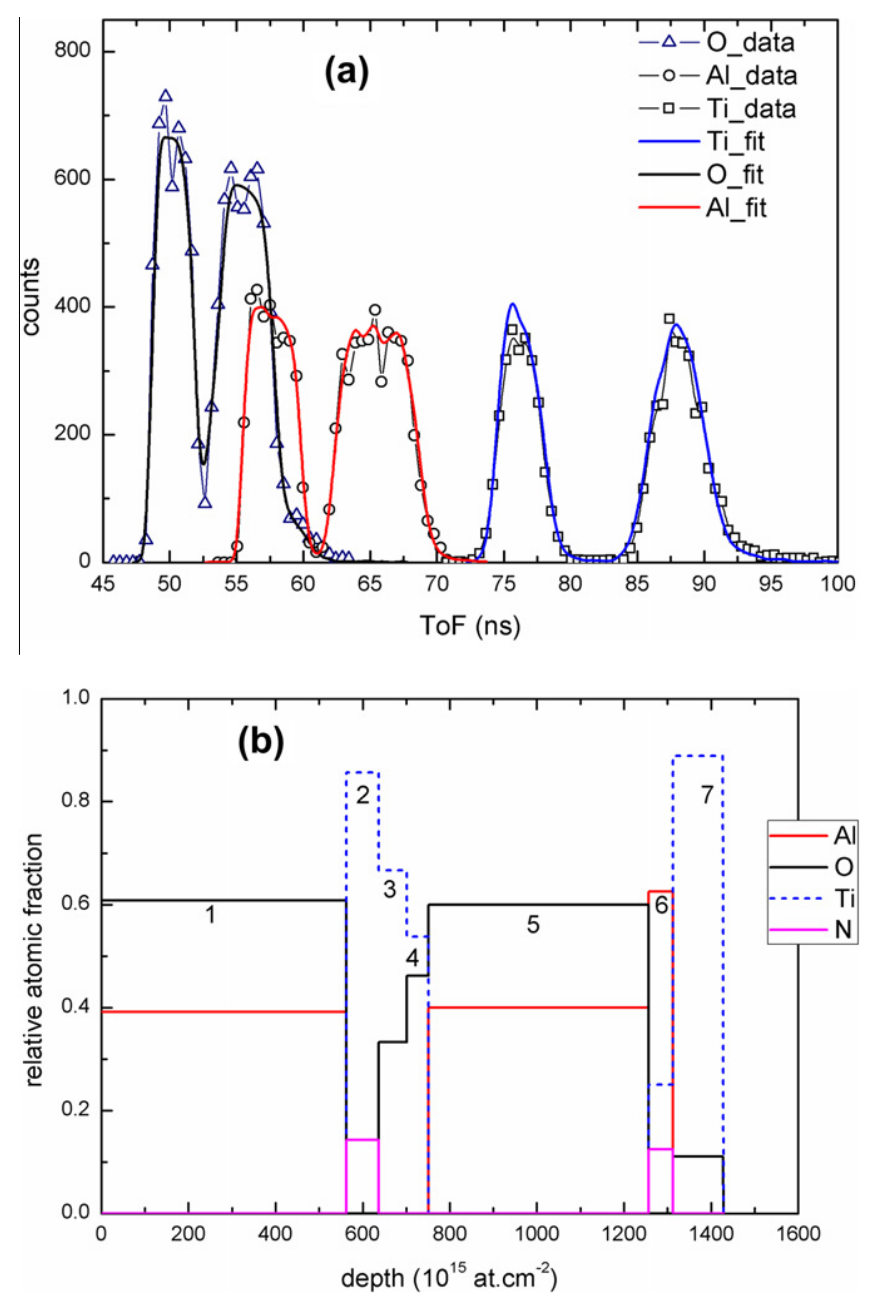

Fig. 8. Experimental and simulated time of flight spectra (a) and the resultant depth profile (b) of the as-deposited sample.

material erosion are shown in Fig. 6. The two superimposed spectra were again taken from an irradiated spot (red) and a virgin region (black), with care taken to compare spectra collected from the same amount of beam dose. The results do not show any difference in the areal densities of the two regions, which suggests that any material removal that may have taken place during the ERDA measurement was insignificant. It should be mentioned also here that the thickness values of the different sub layers obtained through SIMNRA simulation of the RBS spectra were in agreement with the Heavy Ion ERDA values reported in the next subsection.

\subsection{KONZERD calculation of depth profiles}

To get a quantitative comparison of the as-deposited and the annealed layer structures the energy spectra were converted to depth profiles using the energy-to-depth conversion algorithm KONZERD. Prior to that though, an evaluation of the depth resolution at the surface region due to the detector resolution was done to get a rough indication of the energy spread effect of the detector. For instance, for oxygen recoils the energy resolution of the detector system measured from the energy width of the high energy edge surface recoils was found to be $0.17 \mathrm{MeV}$ (or $1.3 \%$ ). This leads to a depth resolution of $64 \times 10^{15}$ at.cm $^{-2}$ calculated using the surface energy approximation [29], or $5.4 \mathrm{~nm}$, assuming $\mathrm{Al}_{2} \mathrm{O}_{3}$ bulk density. A similar calculation for aluminium recoils gives a surface depth resolution of $6.6 \mathrm{~nm}$.
Fig. 7a and $b$ are the calculated depth profiles for the two samples, showing the elemental distributions in the as deposited and annealed samples, respectively. The as-deposited aluminium oxide layers are observed to be largely stoichiometric. It is also interesting to note the location of the nitrogen trace impurity; mostly in the Ti-rich regions - an indication of possible contamination of the Ti source material.

It is not immediately clear however, in Fig. 7a for instance, how much of the tailing of the oxygen profile for example, is due to energy spread effects and how much is due to native oxygen impurity in the Ti target source. Given a surface depth resolution of $64 \times$ $10^{15} \mathrm{at}^{-\mathrm{cm}^{-2}}$ for oxygen atoms, an edge width of about $100 \times 10^{15}$ at.cm ${ }^{-2}$ at the first (topmost) interface seems to sug-gest considerable energy spread effects, notably multiple scatter-ing [30]. Unfortunately with the given limitation in the energy resolution of the detector system, and the fact that other energy spread effects are not taken into account in the calculation, no concrete elucidation of the interfacial structure can be reliably obtained from the depth profiles as they are. This applies to the second interface as well, where it is also not quite possible to say how much of the tailing of the profile edges for the major elements is due to intermixing and how much of it is due to depth resolution limitations. The same can be said for the calculated depth profiles of the annealed sample in Fig. $7 \mathrm{~b}$; where the apparent interfacial reactions can only be confirmed and reliably quantified through a depth profile extraction procedure that takes into account energy
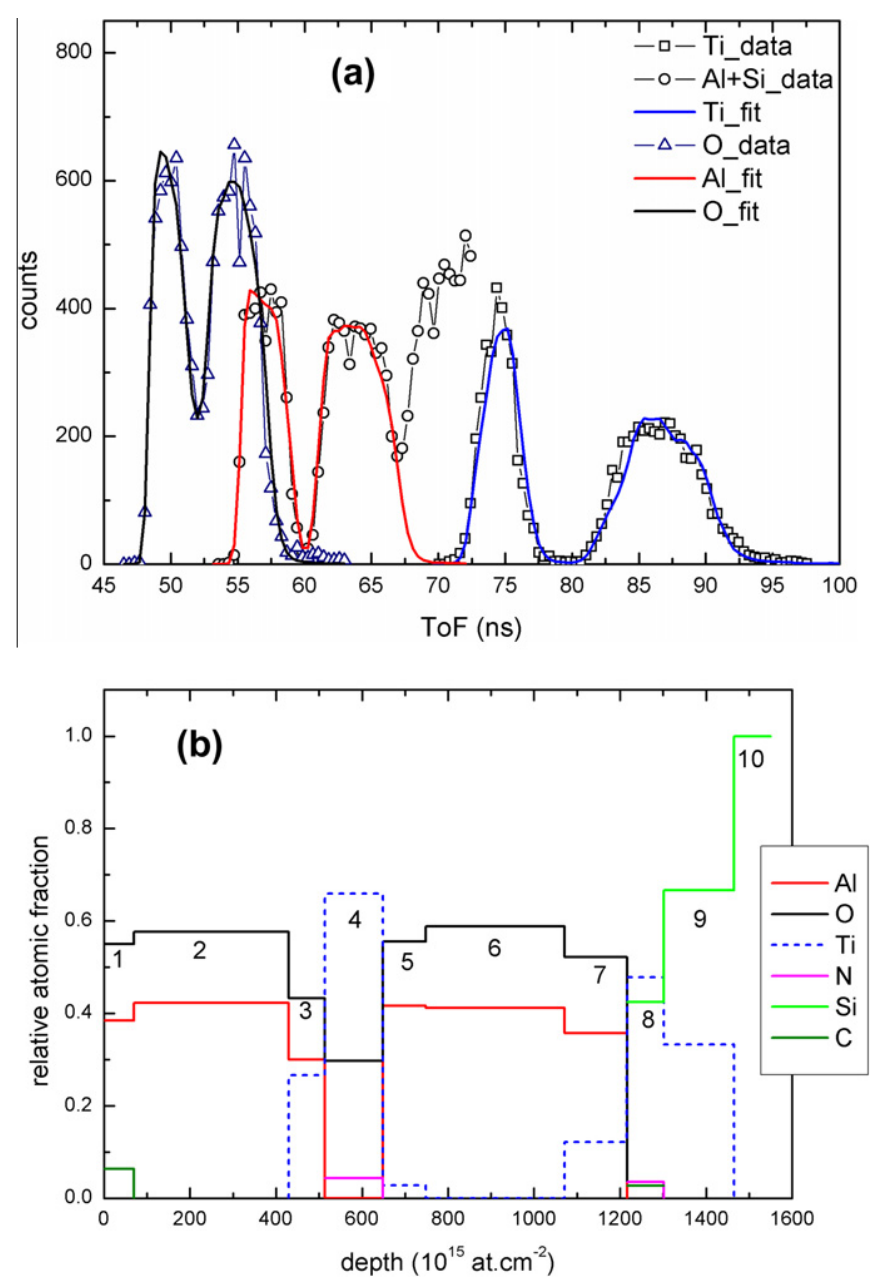

Fig. 9. Experimental and simulated time of flight spectra (a) and the resultant depth profile (b) of the annealed sample. 
spread effects, hence the need for a code such as CORTEO. This output from KONZERD, inconclusive as it seems, does not go to waste as such, as it forms the basis of the input to the simulation code.

\subsection{CORTEO Monte Carlo simulation of depth profiles}

What the KONZERD depth profiles in Fig. 7 provide are intelligible and physically meaningful sample descriptions that are invaluable starting points when it comes to depth profile extraction through simulation. This greatly reduces the risk of assuming wrong sample structures that invariably produce good fits to the experimental spectra. This is aptly demonstrated below in the simulation of the minor elements present as impurities in the multilayer structure, where their location would have been difficult to pinpoint as it were, without the benefit of hindsight from the raw depth profiles.

The CORTEO code permits simulation of either energy or time of flight spectra. Since our energy spectra are derived from time of flight spectra it was decided to simulate the latter. Fig. 8 shows the experimental and simulated elemental ToF spectra of the asdeposited sample, as well as the layer structure giving this 'best' fit. The simulated depth profile shows the average atomic composition plotted as a function of depth into the multilayer film, with the numbered regions indicating sub-layers of varying composition. A similar result for the annealed sample is presented in Fig. 9. Whilst the description of the bulk of the $\mathrm{Al}_{2} \mathrm{O}_{3}$ layers is basi-cally the same in the KONZERD calculated and the CORTEO simu-lated profiles, further information on the interfacial layers can now at least be extracted from the CORTEO depth profiles.

It is now apparent from Fig 8 that the first $\mathrm{Al}_{2} \mathrm{O}_{3}-\mathrm{Ti}$ interface of the as-deposited sample is well defined (regions 1 to 2), whereas the second one (region 6) shows growth of a slight Ti-Al-N interlayer. There is no Al within the first Ti layer (regions 3-5) as Fig. 7a would like to suggest, but rather $\mathrm{N}$ and $\mathrm{O}$ impurities unevenly distributed throughout the layer. The CORTEO simulation also does not suggest any Ti-Si reaction at deposition and so the 'reaction' seen in Fig. 7a could be an energy spread effect. The annealed sample shows considerable intermixing of the $\mathrm{Al}_{2} \mathrm{O}_{3}$ and Ti layers. Closer scrutiny of both the as-deposited and annealed sample profiles reveals that the thickness and stoichiometry of the $\mathrm{Al}_{2} \mathrm{O}_{3}$ layers are largely unchanged after annealing, but the Ti seems to have spread into the $\mathrm{Al}_{2} \mathrm{O}_{3}$ layers (regions 3,5 and 7) and only slightly into the $\mathrm{Si}$ substrate On the other hand, $\mathrm{Si}$ has diffused right through the initial Ti layer, reacting with Ti to form a di-silicide, $\mathrm{TiSi}_{2}$ (region 9). As it happens, in regions of the alumina where there is $\mathrm{Ti}$ the atomic ratio between $\mathrm{Al}$ and $\mathrm{O}$ is still $\sim 2: 3$. This suggests that the dominant diffusing species are Ti atoms. This could be a manifestation of an onset of the Kirkendall effect [31] whereupon the reaction mechanism is through the faster diffusion of Ti atoms into vacancies and other structural defects in the amorphous $\mathrm{Al}_{2} \mathrm{O}_{3}$ layers compared to that of $\mathrm{Al}$ and $\mathrm{O}$ atoms into Ti.

Zalar et al. [24] observed the opposite; $\mathrm{Al}$ and $\mathrm{O}$ diffuse into the metal layer. It should be mentioned though that their investigation focussed on the first 10-20 min of the reaction, at a linear heating rate of $40{ }^{\circ} \mathrm{C} \mathrm{min}-1$, whereas the current study looks at up to 120 min of reaction time at a constant temperature. Indeed, according to Zalar and co-workers, the formation of TiAl phases in the initial stages of the reaction inhibits further transport of oxy-gen at the diffusion front because of lower oxygen solubility in TiAl. This leaves a TiAl-Ti rich diffusion couple at the interfacial re-gion and it is suggested here that it is the presence of this layer that promotes the Kirkendall effect, as also observed by Oliker and Kre-sanov [31] in an $\gamma$-TiAl-Ti coating-substrate composite. The result is that after the formation of TiAl in the initial stages of the reaction Ti moves across the Kirkendall front into the alumina faster than $\mathrm{O}$ and $\mathrm{Al}$ can diffuse into the metal layer, with structural defects occurring a few nanometres before coalescence of the $\mathrm{Al}_{2} \mathrm{O}_{3}$ film providing sinks for the fast diffusing Ti atoms. This classical metallurgical phenomenon [32] has recently been extended by Fan and others [33] to fabricate $\mathrm{ZnAl}_{2} \mathrm{O}_{4}$ spinel nanotubes through a spi-nelforming interfacial solid state reaction of core-shell $\mathrm{ZnO}-\mathrm{Al}_{2} \mathrm{O}_{3}$ nanowires by exploiting the Kirkendall effect. Similarly, the growth of $\mathrm{Si}$ in the second $\mathrm{Ti}$ layer could also be a result of the fas-ter reaction diffusion of Si into the Ti-Si binary phase as Si diffuses 50100 times faster than Ti [34].

\section{Conclusion}

The work reported here introduces the newly set up Heavy Ion ERDA facility at iThemba LABS Gauteng by way of describing a typical application area in the study of multilayer interfacial reactions through thin film depth profiling. The approach used in the study highlights the importance of using both direct calculation and Monte Carlo simulation codes to get the best of both methods to extract more reliable depth profiles. The conclusion drawn from the results is that in an $\mathrm{Al}_{2} \mathrm{O}_{3} / \mathrm{Ti}$ bilayer thin film system annealed in vacuum at $800^{\circ} \mathrm{C}$ for $2 \mathrm{~h}$, after the initial stages of the reaction, the dominant diffusing species become Ti ions, which spread into the alumina faster than $\mathrm{Al}$ and $\mathrm{O}$ can diffuse into the Ti layer, with the structural defects in the topmost layers of the alumina film providing sinks for the Ti ions. This observed interfacial reaction is explained in terms of the Kirkendall effect, a metallurgical phenomenon where unequal diffusion rates of species through an interface lead to a movement of the interface towards the faster moving species.

\section{Acknowledgements}

The authors would like to thank the National Research Foundation (iThemba LABS) and the International Atomic Energy Agency (IAEA) for material and financial support.

\section{References}

[1] D. Xinkang, W. Cong, W. Tianmin, Z. Long, C. Buliang, R. Ning, Microstructure and spectral selectivity of $\mathrm{Mo}-\mathrm{Al}_{2} \mathrm{O}_{3}$ solar selective absorbing coatings after annealing, Thin Solid Films 516 (2008) 3971-3977.

[2] A. Gutiérrez, C. Munuera, M.F. López, J.A. Jiménez, C. Morant, T. Matzelle, N. Kruse, C. Ocal, Surface microstructure of the oxide protective layers grown on vanadium-free Ti alloys for use in biomedical applications, Surf. Sci. 600 (2006) 3780-3784.

[3] W. Mönch, Electronic properties of semiconductor interfaces, Springer Ser. Surf. Sci. 43 (2004).

[4] M. Döbeli, Characterization of oxide films by $\mathrm{MeV}$ ion beam techniques, J. Phys.: Condens. Matter 20 (2008) 264010.

[5] T. Sajavaraa, K. Arstila, Thin film characterisation using MeV ion beams, in: R. Hellborg, H.J. Whitlow, Y. Zhang (Eds.), Ion Beams in Nanoscience and Technology, Springer, 2010. pp. 171-184.

[6] G. Dollinger, A. Bergmaier, T. Faestermann, Elastic recoil detection with single atomic layer depth resolution, Nucl. Instr. Meth. B 136-138 (1998) 603-610.

[7] Z. Siketić, I.B. Radović, M. Jakšić, Development of a time-of-flight spectrometer at the Ruder Bošković Institute in Zagreb, Nucl. Instr. Meth. B 266 (2008) 13281332.

[8] W. Hong, S. Hayakawa, K. Maeda, S. Fukuda, M. Yanokura, M. Aratani, K. kimura, Y. Gohshi, I. Tanihata, Development of a high mass-resolution ToFERDA system for a wide mass range, Nucl. Instr. Meth. B 124 (1997) 95-96.

[9] A. Bergamier, G. Dollinger, C.M. Frey, T. Faestermann, Quantitative elastic recoil detection (ERD), Fresenius. J. Anal. Chem. 353 (1995) 582-584.

[10] M. Mayer, SIMNRA User's Guide, Report IPP 9/113, Max-Planck-Institut für Plasmaphysik, Garching, Germany, 1997.

[11] N.P. Barradas, C. Jeynes, R.P. Webb, Appl. Phys. Lett. 71 (1997) 291.

[12] F. Schiettekatte, Fast Monte Carlo for ion beam analysis simulations, Nucl. Instr. Meth. B 266 (2008) 1880-1885.

[13] K. Arstila, T. Sajavaara, J. Keinonen, Monte Carlo simulation of multiple and plural scattering in elastic recoil detection, Nucl. Instr. Meth. B 174 (2001) $163-172$.

[14] M. Ionescu, Y. Zhao, R. Siegele, D.D. Cohen, E. Stelcer, M. Prior, Heavy ion ToF analysis of oxygen incorporation in $\mathrm{MgB}_{2}$ thin films, Nucl. Instr. Meth. B 266 (2008) 1704-1710. 
[15] W. Bohne, J. Röhrich, G. Röschert, The Berlin time-of-flight ERDA set up, Nucl. Instr. Meth. B 136-138 (1998) 633-637.

[16] R.J. Smith, Y. Zhang, V. Shutthanandan, L.J. Bissel, S. Thevuthasan, W. Jiang, W.J. Weber, NRA and ERDA investigation of helium retention in $\mathrm{SiC}$ as a function of irradiation and annealing, Nucl. Instr. Meth. B 219-220 (2004) 631-635.

[17] S.K. Shretha, H. Timmers, K.S.A. Scott Butcher, M. Wintrebert-Fouquet, Accurate stoichiometric analysis of polycrystalline indium nitride films with elastic recoil detection, Curr. Appl. Phys. A (2004) 237-240.

[18] E. Rauhala, N.P. Barradas, S. Fazinic, M. Mayer, E. Szilàgyi, M. Thompson, Status of ion beam data analysis and simulation software, Nucl. Instr. Meth. B 244 (2006) 436-456.

[19] C.O. Kureba, Energy calibration of the 6 MV EN Tandem accelerator of iThemba LABS Gauteng and measurement of ${ }^{16} \mathrm{O}+{ }^{16} \mathrm{O}$ elastic scattering, MSc thesis, University of the Witwatersrand, Johannesburg, South Africa, 2010.

[20] D.K. Olsen, K.A. Erb, C.M. Jones, W.T. Milner, D.C. Weisser, N.F. Ziegler Calibration of the HHIRF tandem accelerator energy-analyzing magnet, Nucl Instr. Meth. B 254 (1987) 1-6.

[21] S. Ritt, www.midas.psi.ch/htmldoc/index.html, accessed 07/09/2012.

[22] A.M. Kliauga, M. Ferrante, Interface compounds formed during the diffusion bonding of $\mathrm{Al}_{2} \mathrm{O}_{3}$ to Ti, J. Mater. Sci. 35 (2000) 4243-4249.

[23] J.H. Selverian, F.S. Ohuchi, M. Bortz, M.R. Notis, Interface reactions between titanium thin films and (IT2) sapphire substrates, J. Mater. Sci. 26 (1991) 6300-6308.

[24] A. Zalar, B. Baretzky, F. Dettenwanger, M. Rühle, P. Panjan, Interdiffusion at the $\mathrm{Al}_{2} \mathrm{O}_{3} / \mathrm{Ti}$ interface studied in thin film structures, Surf. Interface Anal. 26 (1998) 861-867.
[25] S.M. Meyers et al., Hydrogen interactions with defects in crystalline solids, Rev. Mod. Phys. 64 (1992) 559-617.

[26] J. Jensen, D. Martin, A. Supri, T. Kubart, ERD analysis and modification of $\mathrm{TiO}_{2}$ films with heavy ions, Nucl. Instr. Meth. B 268 (2010) 1893-1898.

[27] P. Trocellier, J. Tirira, P. Massiot, J. Gosset, J.M. Costantini, Nuclear microprobe study of the composition degradation induced in polymides by irradiation with high-energy heavy ions, Nucl. Instr. Meth. B 54 (1991) 118-122.

[28] H.J. Whitlow, A.B. Christina Anderson, C. Sture Peterson, Thermally grown $\mathrm{SiO}_{2}$ film standards for elastic recoil detection analysis, Nucl. Instr. Meth. B 36 (1989) 53-59.

[29] W.K. Chu, J.W. Mayer, M. Nicolet, Backscattering Spectrometry, Academic Press, New York, 1978.

[30] E. Szilágyi, F. Pászti, G. Amsel, Theoretical approximations for depth resolution calculations in IBA methods, Nucl. Instr. Meth. B 100 (1995) 103-121.

[31] V.E. Oliker, V.S. Kresanov, Structure and properties of detonation coatings based on $\gamma$-TiAl, 39, Powder Metall. Met. Ceram. 39 (11-12) (2000) 590-593.

[32] A.D. Smigelskas, E.O. Kirkendall, Zinc diffusion in alpha brass, Trans. AIME 171 (1947) $130-142$.

[33] H.J. Fan, M. Knez, R. Scholz, K. Nielsch, E. Pippel, M. Zacharias, U. Gösele Monocrystalline spinel nanotube fabrication based on the Kirkendall effect, Nat. Mater. 5 (2006) 627-631.

[34] T. Shimozaki, T. Okino, M. Yamane, Y. Wakamatsu, M. Onishi, Effect of impurities on growth of Ti silicides in bulk Ti/Si diffusion couple, Mater. Trans. JIM 38 (10) (1997) 865-870. 